Original Research Paper

\title{
What is Better for Fusion?
}

\author{
Florian Ion Tiberiu Petrescu
}

IFToMM, ARoTMM, Bucharest Polytechnic University, Bucharest, Romania

Article history

Received: 16-03-2020

Revised: 17-04-2020

Accepted: 13-05-2020

Email: fitpetrescu@gmail.com

\begin{abstract}
It is commonly considered that on earth, the fusion reaction could be started more simply if deuterium or more recently tritium, i.e., hydrogen isotopes 2 and 3, are used because they have a larger (and also atomic) nuclear mass and they will be easier to break down and then merge to obtain nuclear energy through fusion. In the present work, the author makes a new hypothesis, contrary to the classical ones, by which it is shown that it could be much simpler to perform the fusion of hydrogen on the ground (like that in the sun) directly on its first protium isotope. Obviously it will work with ions, so for the proposed protium isotope, a proton will be taken into account, so the atom without electron, or in other words only the nucleus of the first hydrogen isotope and not the whole atom.
\end{abstract}

Keywords: Matter, Structure, Dimensions, Nuclear Energy, Fusion, Elementary Particle Dynamics, Condensed Matter

\section{Introduction}

It has long been suspected that a protium fusion is more difficult to achieve than that of the heavier isotopes of hydrogen, due to the fact that the larger masses automatically have larger dimensions and will, therefore, be easier to get closer to each other in order to achieve the fusion for these particles with lower energies (Halliday and Robert, 1966; Kramer, 2011; Krane, 1987; Moses et al., 2009; Shultis and Faw, 2002).

The older theory that larger particles with larger masses could be closer to each other to initiate fusion, stood for a long time and even when we managed to redesign the dimensions of the elementary particles in motion, one did not notice immediately the fact that as they have a larger mass their dimensions diminished will make their proximity even more difficult, so in fact, a closer relationship between two real elementary particles, which are in motion, is easier when they have smaller masses because just then their size increases (Halliday and Robert, 1966; Kramer, 2011; Krane, 1987; Moses et al., 2009; Shultis and Faw, 2002).

One wanted to collect the newly obtained data and put it in a somewhat more orderly way, which was done in the paper (Petrescu and Petrescu, 2019) and then we resumed the energy calculation processes to see which hydrogen isotope is actually more convenient and at this moment, after anticipating new computational relationships that will be presented in the present paper, I immediately noticed that in fact, the protium is the most advantageous for the fusion, because being the smallest as the mass, dynamically it has the largest dimensions and therefore the distances between two such particles are larger than in the case of the other heavier isotopes of hydrogen, which leads to a lower energy requirement to can bring the two elementary particles closer together. It makes the specification essential, even though it is well known by specialists that when we speak of protium (the number 1 isotope of hydrogen) referring to its possible fusion reaction we are referring in fact to the protium ion, the proton, the work with elementary particles in order to achieve the reaction of fusion being made only with positive ions, i.e., with atoms that have lost their electron, in order to enable the acceleration of these elementary particles with the help of a particle accelerator (preferably circular).

In some papers (Halliday and Robert, 1966; Petrescu, 2012a; 2012b; 2012c; 2014; 2018; 2019; Petrescu et al., 2017a; 2017b; 2017c; 2017d; Petrescu and Petrescu, $2018 ; 2019)$ it has been shown that dimensions of atoms and subatomic particles are measured at approximately average values and for low displacement rates of the particles. Dynamically, the dimensions of these particles vary greatly with the variation in their displacement speed. The same thing happens with the energies of these permanently moving particles (Halliday and Robert, 1966; Kramer, 2011; Krane, 1987; Moses et al., 2009; Shultis and Faw, 2002). 


\section{Materials and Methods}

Despite the fact that the authors of the present paper have always considered that fusion on Earth would be easier to achieve than in stars using instead of the first isotope of hydrogen, protium, one of the other two stable isotopes of its, deuterium or tritium, as being with larger masses and therefore easier to split into pieces and then to unite so that we get a stable fusion reaction with higher yields, lately, after reviewing some calculations and related calculation formulas, one had the surprise to find that in fact the first isotope of hydrogen, protium is the easiest to merge, from the point of view of the energy required to start the fusion reaction, because its mass being smaller its radius is actually larger, its dimensions being larger than those of the lower colleagues, deuterium and tritium, which have larger masses, automatically gain smaller dimensions and therefore the energies required to start the fusion reaction will be greater precisely at these lower isotopes, because matter contracts strongly when in motion. If things had happened static, then the hypothesis of an easier merging of the larger masses would have been standing, but since the elementary particles are in a permanent movement, that is, they behave dynamically, they contract with the more their mass is larger, which causes their size to decrease as the masses increase and so the energies needed to approach them for the fusion are greater as their size becomes smaller as their mass increases.

The most well-known isotope of hydrogen is the atom called the protium, which has a single nucleon within its nucleus called a proton (positive charge) or antiproton (with a negative charge) and a single electron or positron orbiting around the nucleus, being a stable particle:

$$
\left\{\begin{array}{l}
R[m]=\frac{h}{2 \pi \cdot \sqrt{K} \cdot c^{2} \cdot M_{0}} \cdot \\
\frac{\sqrt{c^{2}-v^{2}} \cdot \sqrt{2 \cdot c^{2}-2 c \cdot \sqrt{c^{2}-v^{2}}-v^{2}}}{v} \\
J\left[k g \cdot m^{2}\right]=K \cdot M \cdot R^{2} \\
\beta=\frac{v}{c}
\end{array}\right.
$$

The system (1) generates the main relations of calculation of the size of a ray belonging to an elementary particle in motion, relations already presented in the work (Petrescu, 2019) and properly ordered in the work (Petrescu and Petrescu, 2019), where $R$ is the radius of the nucleus, $M_{0}$ the rest mass of the nucleus, $v$ its velocity for that calculation the radius of the nucleus, $h$ is Planck's constant, $c$ the speed of light, $K$ is a constant determined by the mass of rotation of the nucleus, $J$.

For hydrogen the nucleus has a single nucleon, a proton, a single sphere. Mechanical moment of inertia of a sphere around of one of its axes could be determined by using the relationship 2 (Petrescu and Petrescu, 2019):

$\left\{\begin{array}{l}J=\frac{2}{5} \cdot M \cdot R^{2} \\ J=K \cdot M \cdot R^{2}\end{array} \Rightarrow K=\frac{2}{5}\right.$

In order for two elementary particles to be able to be brought closer and closer together, their electrostatic potential energy must be equal to the kinetic energy of such a moving particle with velocity $v$ (relation 3 ), where $R$ is the radius of such an elementary particle, $M$ represents its mass in motion, epsilon ${ }_{0}$ is the permissive constant (the permittivity), while $q_{1}$ and $q_{2}$ are the tasks of the two particles that must be close to each other:

$$
R=\frac{q_{1} \cdot q_{2}}{4 \cdot \pi \cdot \varepsilon_{0} \cdot M \cdot v^{2}}
$$

The angular velocity of rotation of an elementary particle around its own axis is obtained by the expression (4), depending on the mass of the moving particle $M$, its velocity $v$, the speed of light in vacuum $c$ and Plank's constant $h$ :

$$
\omega=\frac{2 \cdot \pi \cdot M \cdot c \cdot v}{h}
$$

The kinetic energy at the rotation of the particle is expressed as a function of its mass at rotation $J$ and the square of its angular velocity $w^{2}$ and using expressions $(4,3)$ one obtains the final version (system 5):

$$
\left\{\begin{array}{l}
E_{c \omega}=\frac{1}{2} \cdot J \cdot \omega^{2} \\
=\frac{1}{2} \cdot K \cdot M \cdot R^{2} \cdot\left(\frac{2 \cdot \pi \cdot M \cdot c \cdot v}{h}\right)^{2} \\
=2 \frac{\pi^{2} \cdot K \cdot c^{2}}{h^{2}} \cdot M^{3} \cdot R^{2} \cdot v^{2} \\
=\frac{K \cdot c^{2} \cdot q_{1}^{2} \cdot q_{2}^{2}}{8 \cdot h^{2} \cdot \varepsilon_{0}^{2}} \cdot M \cdot \frac{1}{v^{2}}
\end{array}\right.
$$

However, the kinetic energy at rotation can be obtained separately with the relativistic relation (6), where it is the one that remains from the total energy of the moving particle after deducting the kinetic energy at translation and its energy at rest:

$E_{c \omega}=M \cdot c^{2}-\frac{1}{2} M \cdot v^{2}-M_{0} \cdot c^{2}$

One identify the two expressions (5) and (6) of the kinetic energy at the rotation of the particle and in this 
way we obtain an equation in $\mathrm{v}^{2}$ of the respective particle (7):

$M \cdot c^{2}-\frac{1}{2} M \cdot v^{2}-M_{0} \cdot c^{2}=\frac{K \cdot c^{2} \cdot q_{1}^{2} \cdot q_{2}^{2}}{8 \cdot h^{2} \cdot \varepsilon_{0}^{2}} \cdot M \cdot \frac{1}{v^{2}}$

One uses Lorentz's relation (8) in order to eliminate the masses from Equation (7) which will thus obtain the aspect (9):

$$
\begin{aligned}
& \frac{M_{0}}{M}=\frac{\sqrt{c^{2}-v^{2}}}{c} \\
& 1-\frac{\sqrt{c^{2}-v^{2}}}{c}=\frac{K \cdot c^{2} \cdot q_{1}^{2} \cdot q_{2}^{2}+4 \cdot v^{2} \cdot h^{2} \cdot \varepsilon_{0}^{2}}{8 \cdot h^{2} \cdot \varepsilon_{0}^{2} \cdot c^{2} \cdot v^{2}}
\end{aligned}
$$

Equation (9) is arranged successively in the forms $(10-12)$ :

$$
1=\frac{K \cdot c^{2} \cdot q_{1}^{2} \cdot q_{2}^{2}+4 \cdot v^{2} \cdot h^{2} \cdot \varepsilon_{0}^{2}+8 \cdot h^{2} \cdot \varepsilon_{0}^{2} \cdot c \cdot v^{2} \cdot \sqrt{c^{2}-v^{2}}}{8 \cdot h^{2} \cdot \varepsilon_{0}^{2} \cdot c^{2} \cdot v^{2}}
$$

$8 \cdot h^{2} \cdot \varepsilon_{0}^{2} \cdot c^{2} \cdot v^{2}=$

$K \cdot c^{2} \cdot q_{1}^{2} \cdot q_{2}^{2}+4 \cdot v^{2} \cdot h^{2} \cdot \varepsilon_{0}^{2}+8 \cdot h^{2} \cdot \varepsilon_{0}^{2} \cdot c \cdot v^{2} \cdot \sqrt{c^{2}-v^{2}}$

$$
\frac{2 \cdot c^{2}-1}{2 \cdot c}-\frac{K \cdot c \cdot q_{1}^{2} \cdot q_{2}^{2}}{8 \cdot h^{2} \cdot \varepsilon_{0}^{2} \cdot v^{2}}=\sqrt{c^{2}-v^{2}}
$$

Equation (12) in $v^{2}$ rises to the square and is arranged in the form (13):

$$
\begin{aligned}
& \left(v^{2}\right)^{3}+\frac{1-4 c^{2}}{4 c^{2}} \cdot\left(v^{2}\right)^{2}-\frac{q_{1}^{2} \cdot q_{2}^{2} \cdot K \cdot\left(2 c^{2}-1\right)}{8 \cdot h^{2} \cdot \varepsilon_{0}^{2}} \cdot v^{2} \\
& +\frac{\left(q_{1} \cdot q_{2}\right)^{4} \cdot c^{2} \cdot K^{2}}{64 \cdot h^{4} \cdot \varepsilon_{0}^{4}}=0
\end{aligned}
$$

Solving the equation of degree 6 in $v$ or degree 3 in $v^{2}$, one obtains the real solution $v^{2}$ and implicitly $\mathrm{v}$, depending on $K$.

\section{Results and Discussion}

For the proton, the following values are listed in Table 1.

The second stable isotope of hydrogen is the atom called deuterium, an atom that in its nucleus possesses two nucleons, a proton (positive charge) or antiproton (with a negative charge) and a neutron (no charge) or an antineutron and obviously the atom has and a single electron or positron orbiting around the nucleus. Its nucleus, which can be accelerated when trying to obtain a fusion reaction, is called deuteron (Fig. 1).

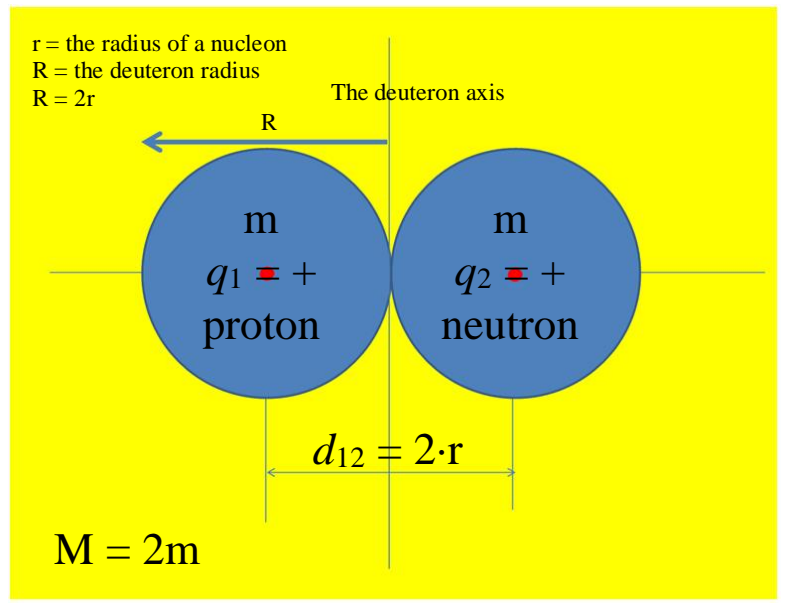

Fig. 1: A deuteron

Table 1: The proton values

\begin{tabular}{ll}
\hline \multicolumn{2}{c}{ Proton } \\
\hline Kproton & 0.4 \\
$\varepsilon_{0}\left[\mathrm{C}^{2} / \mathrm{Nm}^{2}\right]$ & $8.85419 \mathrm{E}-12$ \\
$\mathrm{c}[\mathrm{m} / \mathrm{s}]$ & 299800000 \\
$\mathrm{~h}[\mathrm{Js}]$ & $6.626 \mathrm{E}-34$ \\
$\mathrm{q}[\mathrm{C}]$ & $1.602 \mathrm{E}-19$ \\
$\mathrm{~m} 0$ proton $[\mathrm{kg}]$ & $1.67262 \mathrm{E}-27$ \\
$\mathrm{~m}_{0}$ deuteron $[\mathrm{kg}]$ & $3.34524 \mathrm{E}-27$ \\
$\mathrm{RAD}=\sqrt{c^{2}-v^{2}}[\mathrm{~m} / \mathrm{s}]$ & 299799202.1 \\
$\mathrm{v}[\mathrm{m} / \mathrm{s}]$ & 691664.1699 \\
$\mathrm{v}^{2}\left[\mathrm{~m}^{2} / \mathrm{s}^{2}\right]$ & $4.78399 \mathrm{E}+11$ \\
$\mathrm{R}[\mathrm{m}]$ & $3.8357 \mathrm{E}-19$ \\
$\mathrm{U}=\mathrm{Ep}[\mathrm{J}]$ & $3.00671 \mathrm{E}-10$ \\
$\mathrm{U}=\mathrm{Ep}[\mathrm{eV}]$ & 1876789192 \\
$\mathrm{U}=\mathrm{Ep}[\mathrm{KeV}]$ & 1876789.192 \\
$\mathrm{U}=\mathrm{Ep}[\mathrm{MeV}]$ & 1876.789192 \\
$\mathrm{U}=\mathrm{Ep}[\mathrm{GeV}]$ & 1.876789192 \\
\hline
\end{tabular}

Table 2: The deuteron values

\begin{tabular}{ll}
\hline \multicolumn{2}{c}{ Deuteron } \\
\hline Kdeuteron & 0.35 \\
$\varepsilon_{0}\left[\mathrm{C}^{2} / \mathrm{Nm}^{2}\right]$ & $8.85419 \mathrm{E}-12$ \\
$\mathrm{c}[\mathrm{m} / \mathrm{s}]$ & 299800000 \\
$\mathrm{~h}[\mathrm{Js}]$ & $6.626 \mathrm{E}-34$ \\
$\mathrm{q}[\mathrm{C}]$ & $1.602 \mathrm{E}-19$ \\
$\mathrm{~m}$ proton $[\mathrm{kg}]$ & $1.67262 \mathrm{E}-27$ \\
$\mathrm{~m}_{0}$ deuteron $[\mathrm{kg}]$ & $3.34524 \mathrm{E}-27$ \\
$\mathrm{RAD}=\sqrt{c^{2}-v^{2}}[\mathrm{~m} / \mathrm{s}]$ & 299799301.9 \\
$\mathrm{v}[\mathrm{m} / \mathrm{s}]$ & 646992.5338 \\
$\mathrm{v}^{2}\left[\mathrm{~m}^{2} / \mathrm{s}^{2}\right]$ & $4.18599 \mathrm{E}+11$ \\
$\mathrm{R}[\mathrm{m}]$ & $1.91787 \mathrm{E}-19$ \\
$\mathrm{U}=\mathrm{Ep}[\mathrm{J}]$ & $6.01336 \mathrm{E}-10$ \\
$\mathrm{U}=\mathrm{Ep}[\mathrm{eV}]$ & 3753539016 \\
$\mathrm{U}=\mathrm{Ep}[\mathrm{KeV}]$ & 3753539.016 \\
$\mathrm{U}=\mathrm{Ep}[\mathrm{MeV}]$ & 3753.539016 \\
$\mathrm{U}=\mathrm{Ep}[\mathrm{GeV}]$ & 3.753539016 \\
\hline
\end{tabular}

The constant $K$ is determined from the system (14): 


$$
\left\{\begin{array}{l}
J=\frac{7}{5} \cdot M \cdot r^{2}=\frac{7}{5} \cdot M \cdot \frac{R^{2}}{4}=\frac{7}{20} \cdot M \cdot R^{2} \\
J=K \cdot M \cdot R^{2}
\end{array} \Rightarrow K=\frac{7}{20}\right.
$$

For the deuteron, the following values are listed in Table 2 (Halliday and Robert, 1966; Kramer, 2011; Krane, 1987; Moses et al., 2009; Shultis and Faw, 2002):

\section{Conclusion}

As can be seen from the two exposed tables, the radius of a deuteron is almost half that of a proton, which makes its energy of starting the fusion reaction almost double compared to that required by the proton (obvious for the same purpose).

Because one does not yet have the temperature conditions of the sun and the stars, to be able to produce hot fusion only at temperatures of tens of billions of degrees, we have to try to make here on Earth a fusion reaction based on accelerated particles at the energy required to initiate the fusion reaction, for which a torus-like nuclear reactor is needed, similar to a donut or a barrel. Obviously, this reaction can be carried out cold or hot (in order to gain some more free kinetics for the fusion particles).

Contrary to classical expectations and assumptions, the paper indicates that it would be easier to start the fusion reaction to the protium nucleus, its ion, that is to say, a proton, than to continue to use deuterium as fuel, like until now, because by ionization to obtain the deuterium nuclei (ions), i.e., deuterons, one needs then more energy per deuteron before colliding them.

However, the required energy values given in $\mathrm{GeV}$ are much higher in both variants compared to those of some $\mathrm{keV}$ predicted by the classical, static assumptions, where the calculations also showed the fusion order indicating deuterium as being more suitable than protium.

Considering that the hydrogen in its first isotope can be very easily obtained by various industrial methods, it is simple to try to obtain the fusion with protons, the cheap, abundant and sustainable raw material being as friendly as the respective reaction, which is it can easily control permanently through the level of the accelerated particles introduced into the reaction vessel, a level that can be raised or lowered as needed, or stopped quickly when the reaction is trying to get out of control.

\section{Acknowledgment}

The author acknowledges INIS for his research in the field.

\section{Ethics}

This article is original and contains unpublished material. Author declares that are not ethical issues and no conflict of interest that may arise after the publication of this manuscript.

\section{References}

Halliday, D. and R. Robert, 1966. Physics, Part II. 1st Edn., John Wiley and Sons, Inc., New York.

Kramer, D., 2011. DOE looks again at inertial fusion as a potential clean-energy source. Phys. Today, 64: 26-26. DOI: 10.1063/1.3563814

Krane, K.S., 1987. Introductory Nuclear Physics. 3rd Edn., Wiley and Sons, New York, ISBN-10: $047180553 \mathrm{X}, \mathrm{pp}: 864$.

Moses, E.I., R.N. Boyd, B.A. Remington, C.J. Keane and R. Al-Ayat, 2009. The national ignition facility: Ushering in a new age for high energy density science. Phys. Plasmas, 16: 041006-041006. DOI: $10.1063 / 1.3116505$

Petrescu, F.I.T., 2014. Nuclear fusion. Infinite Energy, 20: 44-47.

Petrescu, F.I.T., 2012a. Cold Nuclear Fusion. 1st Edn., BoD - Books on Demand, ISBN-10: 3848228521, pp: 102.

Petrescu, F.I.T., 2012b. Cold Nuclear Fusion. 1st Edn., Create Space Publisher, USA, ISBN-13: 978-1-4782-3426-5, pp: 80.

Petrescu, F.I.T., 2012c. A New Atomic Model. Books On Demand, ISBN-13: 978-3848218943, pp: 62.

Petrescu, R.V., R. Aversa, S. Li, R. Bucinell and S. Kosaitis et al., 2017a. Electron dimensions. Am. J. Eng. Applied Sci., 10: 584-602. DOI: 10.3844/ajeassp.2017.584.602

Petrescu, R.V., R. Aversa, S. Kozaitis, A. Apicella and F.I.T. Petrescu, 2017b. Deuteron dimensions. Am. J. Eng. Applied Sci., 10: 649-654. DOI: 10.3844/ajeassp.2017.649.654

Petrescu, R.V., R. Aversa, S. Kozaitis, A. Apicella and F.I.T. Petrescu, 2017c. Some proposed solutions to achieve nuclear fusion. Am. J. Eng. Applied Sci., 10: 703-708. DOI: 10.3844/ajeassp.2017.703.708

Petrescu, R.V., R. Aversa, S. Kozaitis, A. Apicella and F.I.T. Petrescu, 2017d. Some basic reactions in nuclear fusion. Am. J. Eng. Applied Sci., 10: 709-716. DOI: 10.3844/ajeassp.2017.709.716

Petrescu, F.I.T., 2019. About the nuclear particles' structure and dimensions. Comp. Part. Mech., 6: 191-194. DOI: 10.1007/s40571-018-0206-7

Petrescu, F.I.T., 2018. About the triton structure. Am. J. Eng. Applied Sci., 11: 1293-1297. DOI: 10.3844/ajeassp.2018.1293.1297

Petrescu, N. and F. Petrescu, 2018. Elementary structure of matter can be studied with new quantum computers. Am. J. Eng. Applied Sci., 11: 1062-1075. DOI: 10.3844/ajeassp.2018.1062.1075 
Petrescu, F.I.T. and R.V.V., Petrescu, 2019. Nuclear hydrogen structure and dimensions. Int. J. Hydrogen Energy, 44: 10833-10837.

DOI: 10.1016/j.ijhydene.2019.02.140

Shultis, J.K. and R.E. Faw, 2002. Fundamentals of Nuclear Science and Engineering. CRC Press, New York, ISBN-10: 0824708342, pp; 520.

\section{Nomenclature}

$\Rightarrow$ The Planck constant: $\mathrm{h}=6.626$ E-34 [Js]

q $\quad \Rightarrow$ Electrical elementary load: qe $=-1.6021$ $\mathrm{E}-19[\mathrm{C}] \mathrm{qp}=+1.6021 \mathrm{E}-19[\mathrm{C}]$

c $\quad=$ The light speed in vacuum: $\mathrm{c}=2.997925$ $\mathrm{E}+08[\mathrm{~m} / \mathrm{s}]$

The permissive constant (the permittivity):

$$
\varepsilon_{0}=8.85418 \cdot 10^{-12}\left[\frac{C^{2}}{N \cdot m^{2}}\right]
$$

$\mathrm{n}=$ The principal quantum number (the Bohr quantum number);

$\mathrm{Z}=$ The number of protons from the atomic nucleus (the atomic number);

$\mathrm{m} 0[\mathrm{~kg}] \quad \Rightarrow$ The rest mass of one particle

m0electron $=9.11 \mathrm{E}-31[\mathrm{~kg}]$

m0proton $=1.672621898(21) \mathrm{E}-27[\mathrm{~kg}]$

m0neutron $=1.674927471(21) \mathrm{E}-27[\mathrm{~kg}]$

m0deuteron $=3.34449 \mathrm{E}-27[\mathrm{~kg}]$

m0triton $=5.00827 \mathrm{E}-27[\mathrm{~kg}]$ 